\title{
The Arab Astronomical Society (ArAS): Developing Astrophysics Research in the Arab World
}

\section{Zouhair Benkhaldoun ${ }^{1,6}$, Raid M. Suleiman ${ }^{2,6}$, Ismael Moumen ${ }^{3,6}$, Moza M. Al-Rabban ${ }^{4}$ and Randa Asa'd ${ }^{5,6}$}

\author{
${ }^{1}$ Oukaimeden Observatory, Caddi Ayyad University, LPHEA, Faculté des Sciences Semlalia, \\ Marrakesh, Morocco \\ ${ }^{2}$ Harvard-Smithsonian Center for Astrophysics, Cambridge, MA, USA \\ ${ }^{3}$ Université Laval, Québec, Canada \\ ${ }^{4}$ Arab Scientific Community Organization, Doha, Qatar \\ ${ }^{5}$ American University of Sharjah, Sharjah, United Arab Emirates \\ ${ }^{6}$ Arab Astronomical Society, Caddi Ayyad University, Marrakesh, Morocco \\ email: info@ar-as.org
}

\begin{abstract}
The Arab Astronomical Society (ArAS) was officially created during the constitutional assembly held in Marrakech (Morocco) on November 30, 2016, and legally recognized on May 15, 2017. ArAS is composed of a group of Arab researchers and students in the field of astrophysics who aim to develop research in this field in the Arab world (22 countries). ArAS is working on bridging the gap between the Arab astrophysicists in the Arab world and those around the world by organizing collaborative workshops and international scientific meetings, offering scholarships and developing graduate programs in astrophysics. Presently, the Society is working on establishing personal and material scientific infrastructure in the Arab world by training advanced undergraduate and graduate students in astrophysics and stimulating the building of new telescopes on the best sites in the Arab world. This will be accomplished through the hosting of specialized schools and conferences in astrophysics, international collaborations, facilitation of students' and post-docs' training in international research centres and universities, the establishment of prizes in astronomy to honour leading Arab scientists in astronomy and to motivate junior researchers to present notable works in astronomy. In this work, we present the on-going ArAS activities as well as future projects. ArAS is a young but energetic organization which is welcoming collaborations and partnership with other groups.
\end{abstract}

Keywords. Arab Astronomical Society, ArAS, astronomy development

\section{Introduction}

Astronomy is one of the oldest fields of science. Throughout the years, different societies have made different contributions to the development of this field. The contribution of Arabs and Muslims is well known. From the names of the stars that still have their Arabic origin to the invention and development of astronomical devices like the astrolabe. Today, however, the contribution of the Arab world is not as significant as it used to be (Al-Naimiy 2011; Guessoum 2013). Although there are some great initiatives across the Arab countries, the overall status is much less than desired (Pović et al. 2018; Benkhaldoun 2018). Figure 1 shows the papers published by various Arab countries and the percentage of each country's contribution. With the aim of improving these numbers by advancing astrophysics research in the region, the Arab Astronomical Society (ArAS) was founded. 


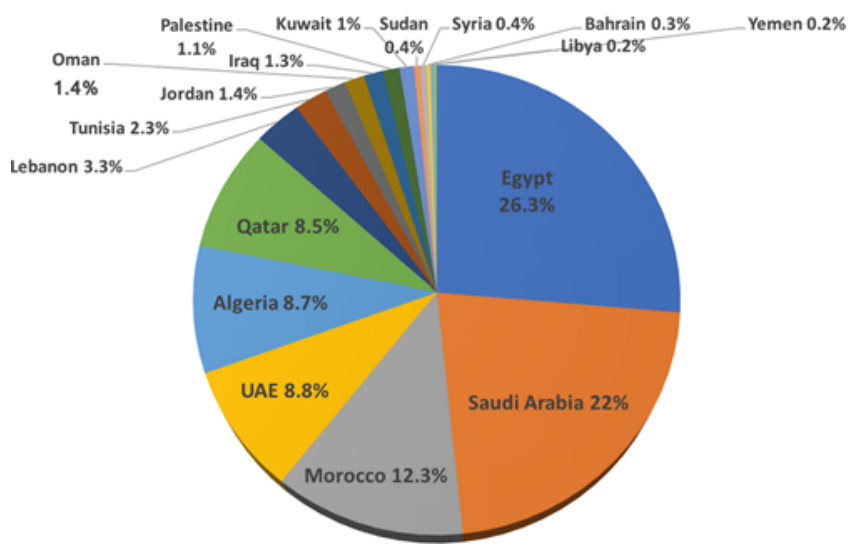

Figure 1. Distribution of scientific contribution for each Arab country - Source: Web of Science.

\section{What is ArAS?}

The Arab Astronomical Society (ArAS) is a professional not-for-profit organization that aims to advance professional astronomy in the region. Its primary objective is to stimulate education and scientific research in the field of astronomy in the Arab countries. It is intended to be the assembly where ideas and initiatives can be presented to national, regional and international institutions. ArAS was established in November 2016 in Marrakesh, Kingdom of Morocco. The formal and legal recognition became official in May 2017. It consists of a group of professional Arab researchers and students in the field of astrophysics who aim to develop astrophysics research and education in the Arab world.

\section{Current ArAS Activities}

\subsection{ArAS Schools for Astrophysics (ArAS SfA)}

In 2016, the ArAS obtained an IAU-OAD grant to organise its First School for Astrophysics (ArAS SfA) with the goal of training interested Arab students by renowned Arab and international professional astronomers. Students got the opportunity to learn and network with them. It was an excellent opportunity to get students exposed to local and international on-going research activities.

This First SfA School took place at Caddi Ayyad University in Marrakesh, Morocco in the period November 28 to December 3, 2016. Full financial support was given to 12 students from the Arab countries in addition to partial support for other students based on the financial needs and scientific merit of the candidates. The distribution of students by gender for this school is shown in Figure 2. It covered topics ranging from exoplanets, solar physics, stellar clusters, cosmology, observational astronomy and technical tools, presented by speakers from USA, Canada, UK, France, Morocco, Algeria and the UAE.

The Al Akhawayn University in Ifrane hosted the Second SfA school in Morocco on November 19-25, 2017 (Fig. 3). Full financial support for nine students from the Arab countries was available in addition to partial support for four students. The themes were more focused on the following pillars: (1) Theory for three main topics (solar physics, exoplanets and star clusters); (2) Writing a telescope proposal; (3) Observations; (4) Data reduction analysis; and (5) Writing a paper. They were presented by speakers from USA, Canada, Germany, Italy, France, Morocco, Lebanon and the UAE. 


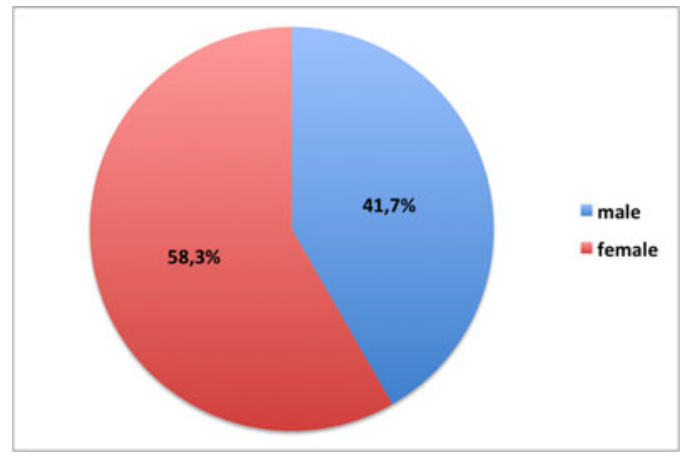

Figure 2. Distribution of students participating at the First ArAS SfA Winter School.

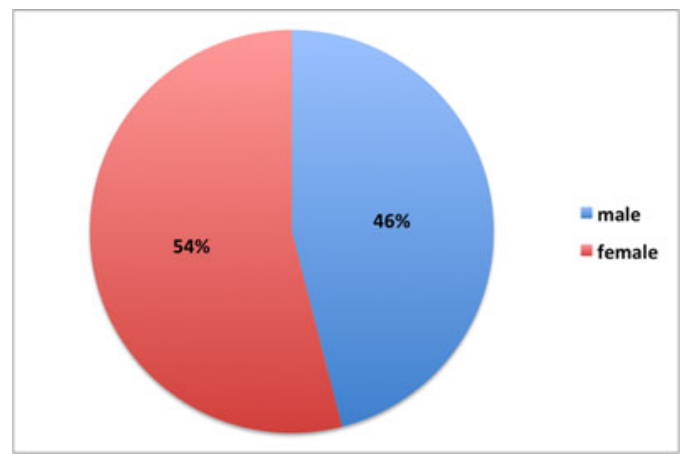

Figure 3. Distribution of students participating at the Second ArAS SfA Winter School.

The third school is going to take place at the American University of Beirut, Lebanon in the period November 12-18, 2018.

Al Katameya Observatory was chosen to host the fourth edition of ArAS SfA in 2019.

\subsection{Scholarships for students and professors}

ArAS provides funds to support students and professors from the Arab World. Currently, ArAS is offering one scholarship (Al-Meeraj) which is dedicated to Palestinian students interested in an undergraduate degree in astronomy with the goal of pursuing graduate studies in astronomy.

\subsection{ArAS Visitor Program}

ArAS offers an important opportunity to the students, researchers and professors from Arab countries to do professional research in high-level centres of research and universities. Since 2016, ArAS facilitated the visit of Arab professionals and four students from different Arab countries to the Harvard-Smithsonian Center for Astrophysics to conduct research. Up to the present date, the outcome of these visits is positive. For example, a paper was published in the Astrophysical Journal as a result of a collaboration that started during the visit; more papers are in progress. Another significant benefit of the visitor programme is obtaining telescope time to observe with both the SOAR and Magellan telescopes. 


\section{ArAS Activities in Progress}

\subsection{The ArAS Mobile Observatory (ArAS MO)}

ArAS MO aims to develop the technical and professional skills in observational astronomy for students and professors in different Arab countries (especially those who do not have access to a professional telescope) by creating an Astronomical Mobile Observatory. ArAS MO will be used by any interested university in the Arab World for the training of students and professors in astronomy. It will also be used to obtain data with a publication quality allowing for various astronomical research projects. Additionally, it will be an essential tool during the next years to help in the efforts of building new groundbased observatories in the Arab World by using ArAS MO in site testing. This project is currently underway and the first light of the ArAS MO is expected in December 2019, during the meeting Astronomical Site Selection for Africa and the Middle East region.

\subsection{Scientific Conferences}

The ArAS Meeting will be held every three years during which the General Assembly is organized. During the ArAS Meetings, students, researchers and professors from the Arab world and outside the Arab World will find a unique opportunity to share their results with their collaborators in the Arab World. The first ArAS meeting will be held in late 2019 in parallel with the organisation of Telescopes in Africa and the Arab World workshop.

\section{Acknowledgements}

We would like to thank Andrew Szentgyorgyi and Kelly Chance for their continuous support. The First and the Third Arab School for Astrophysics were supported by the IAU/OAD grants P161FAWSA and P181ArASSfA-3. The First and Second Arab Winter School for Astrophysics were supported by the Smithsonian Astrophysical Observatory, Al Akhawayn University and the Arab Scientific Community Organization. The Oukaimeden Observatory and Cadi Ayyad University have provided constant support to all ArAS activities.

\section{References}

Al-Naimiy, H. M. K. 2011, in The role of Astronomy and Society and Culture, D. Valls-Gabaud \& A. Boksenberg (eds), IAU Symposium 260, 429-437 (Cambridge: Cambridge University Press).

Benkhaldoun, Z. 2018, Nature Astronomy, 2, 352-354

Guessoum, N. 2013, Nature, 498, 161-164

Pović, M. et al., 2018, Nature Astronomy, 2, 507-510 